\title{
Predictive value of the modified Glasgow Prognostic Score for the therapeutic effects of molecular-targeted drugs on advanced renal cell carcinoma
}

\author{
HIROFUMI OHMURA ${ }^{1}$, KEITA UCHINO ${ }^{1}$, TATSUHIRO KAJITANI ${ }^{1}$, NAOTAKA SAKAMOTO ${ }^{2}$ and EISHI BABA ${ }^{3}$ \\ Departments of ${ }^{1}$ Medical Oncology and ${ }^{2}$ Urology, Clinical Research Institute, National Hospital Organization \\ Kyushu Medical Center, Fukuoka 810-8563; ${ }^{3}$ Department of Comprehensive Clinical Oncology, \\ Faculty of Medical Sciences, Kyushu University, Fukuoka 812-0054, Japan
}

Received May 11, 2016; Accepted September 23, 2016

DOI: $10.3892 / \mathrm{mco} .2017 .1205$

\begin{abstract}
Inflammation is considered to be a prognostic factor for renal cell carcinoma (RCC). An inflammation-based prognostic score (modified Glasgow Prognostic Score; mGPS) is widely used for preoperative patients; however, little information is available regarding its prognostic value in patients with RCC treated with molecular-targeted drugs. A total of 32 advanced and recurrent RCC patients initially treated with molecular-targeted drugs from October, 2009 to August, 2015 were retrospectively investigated. Information on patient characteristics prior to treatment initiation and the clinical course were retrieved from clinical records. The correlation between survival and patient variables was analyzed. Survival was compared among patient groups according to the mGPS score. The median patient age was 66 years. The percentage of patients with an Eastern Cooperative Oncology Group performance status of 0 or 1 was 87.5 , and $65.6 \%$ of the RCCs were clear cell carcinomas. A Memorial Sloan-Kettering Cancer Center index of good or intermediate was determined for $75 \%$ of the patients. Sunitinib, pazopanib or sorafenib was administered to 56, 22 and $13 \%$ of the cases, respectively. An mGPS score of 0,1 and 2 was calculated for 66,9 and $25 \%$ of the cases, respectively. Patients in the mGPS low group (score 0) exhibited significantly better progression-free survival (PFS) and overall survival (OS) compared with patients in the mGPS high group (score 1 or 2) (median PFS, 307 vs. 70 days and median OS, 1,081 vs. 140 days, respectively). In conclusion, inflammatory status as assessed by the mGPS score was
\end{abstract}

Correspondence to: Dr Keita Uchino, Department of Medical Oncology, Clinical Research Institute, National Hospital Organization Kyushu Medical Center, 1-8-1 Jigyouhama, Chuou-ku, Fukuoka 810-8563, Japan

E-mail:keitauch@kyumed.jp

Key words: renal cell carcinoma, inflammation, chemotherapy, modified Glasgow Prognostic Score, survival closely associated with the prognosis of RCC patients treated with molecular-targeted therapy.

\section{Introduction}

Kidney cancer accounts for $\sim 4 \%$ of all cancers worldwide and renal cell carcinoma (RCC) is the most common type of kidney cancer (1-3). Radical nephrectomy is usually performed for advanced RCC; however, recurrence with distant metastasis occurs in $>25 \%$ of the patients (4) and chemotherapy is employed for these metastatic RCC (mRCC) patients. While interleukin (IL)-2 and interferon (IFN)- $\alpha$ have been used for mRCC $(5,6)$, molecular-targeted drugs, such as multi-kinase inhibitors, have been recently developed and exhibit great efficacy for mRCC, resulting in improved survival (7-9). In particular, a pivotal study that investigated sunitinib treatment demonstrated a median overall survival of 32 months for mRCC (10). The current standard therapy for mRCC is thus the first-line use of these molecular-targeted drugs.

To predict the prognosis of $\mathrm{mRCC}$ patients treated with IFN- $\gamma$, the Memorial Sloan-Kettering Cancer Center (MSKCC) index, which includes measurements of performance status, serum lactate dehydrogenase level, serum calcium level, hemoglobin and time interval from diagnosis to therapy, was established (11). The Heng model was proposed for predicting the prognosis of $\mathrm{mRCC}$ patients who were treated with anti-angiogenesis inhibitors and the parameters assayed included neutrophil and platelet counts $(12,13)$. The appropriateness of the index used is based on the acknowledgement of the close association between the clinical course of mRCC and inflammatory status. Increased serum levels of inflammatory cytokines in RCC patients have been reported (14-16), whereas other parameters associated with inflammatory conditions include not only fever, erythrocyte sedimentation rate and C-reactive protein (CRP) levels (17), but also neutrophil and lymphocyte counts (18-21).

Inflammatory cytokines, such as IL-1 $\beta$ and IL-6, are known to induce systemic inflammation, alteration of systemic metabolism, atrophy of muscle and fat tissue and loss of appetite and body weight, resulting in cancer cachexia. A strong association between inflammatory status and cachexia 
has also been reported in cancers of the stomach, lung and pancreas (22-26).

One of the inflammation-based prognostic indices, the modified Glasgow Prognostic Score (mGPS), is a simple scoring system that is calculated based on serum CRP and albumin levels and has been widely used as a predictive tool for mRCC (27-29). Since the current therapy for mRCC is monotherapy with molecular-targeted agents, determination of the association between the efficacy of these therapies and inflammatory conditions is required. However, the prognostic value of mGPS in mRCC patients treated with molecular-targeted drugs remains unclear. In the present study, we retrospectively investigated the mGPS and the therapeutic effects of molecular-targeted drugs in Japanese patients with advanced $\mathrm{RCC}$ in a single institution.

\section{Patients and methods}

Patients. A total of $45 \mathrm{RCC}$ patients who were treated at the Department of Medical Oncology, Clinical Research Institute, National Hospital Organization Kyushu Medical Center (Fukuoka, Japan) between October, 2009 and August, 2015 were screened. Of these patients, 32 with histologically and/or radiographically confirmed advanced $\mathrm{RCC}$ who were treated with a molecular-targeted drug as first-line therapy were investigated. The eligibility criteria were as follows: Measurable or evaluable tumor lesions according to the Response Evaluation Criteria in Solid Tumors guidelines, version 1.1 (https://ctep .cancer.gov/protocoldevelopment/docs/recist_guideline.pdf), confirmation of the date of death or progressive disease, and no evidence of active infection. There was no restriction regarding Eastern Cooperative Oncology Group (ECOG) performance status (PS) or previous cytokine therapies. This study was approved by the Ethics Committee of the National Organization Kyushu Medical Center and was conducted according to the guidelines for biomedical research specified in the Declaration of Helsinki. Due to the retrospective nature of the present study, patient informed consent was not obtained.

Clinical variables assessed. Information on patient characteristics and clinical data were retrieved from medical records and retrospectively reviewed. The items surveyed in this study included age, gender, PS, primary tumor site, histopathological diagnosis, metastatic and recurrent sites, tumor status and previous renal surgery. Information regarding systemic chemotherapy included the chemotherapy regimen, progression-free survival (PFS), overall survival (OS), reasons for terminating initial chemotherapy and subsequent therapies. Information associated with inflammatory conditions included the results of blood tests (white blood cells, neutrophils, lymphocytes, hemoglobin, platelets, albumin and CRP) and the mGPS. All data corresponded to the period within 7 days prior to chemotherapy initiation.

The mGPS consists of two factors, namely ther serum levels of CRP (normal range, 0.00-0.30 mg/dl) and albumin (normal range, 4.0-5.0 g/dl) (30). Patients with an increment in the CRP level $(>1.0 \mathrm{mg} / \mathrm{dl})$ and a decrease in the albumin level $(<3.5 \mathrm{~g} / \mathrm{dl})$ were assigned a score of 2 , those with only an increment in the CRP level ( $>1.0 \mathrm{mg} / \mathrm{dl})$ were assigned a score of 1 , and those with a normal serum CRP level were assigned a score of 0 .

Statistical analysis. PFS was defined as the period from the initiation of therapy to the day of tumor progression or death from any cause. OS was defined as the period from initiation of therapy to the day of death from any cause. The Kaplan-Meier method was used to estimate PFS and OS, and the log-rank test was used to compare the two groups according to the mGPS. Correlations between survival and clinicopathological characteristics were analyzed by using Wilcoxon's test and Pearson's Chi-square test. $\mathrm{P}<0.05$ was considered to indicate statistically significant differences. All the analyses were performed using SPSS software, version 21 (IBM SPSS, Armonk, NY, USA).

\section{Results}

Patient characteristics. The median age of the 32 patients was 66 years (range, 33-82 years) (Table I); 20 men (62.5\%) and 12 women $(37.5 \%)$ were included. In terms of histological diagnosis of the renal tumor, clear cell carcinoma was diagnosed in 21 patients $(65.5 \%)$; papillary or sarcomatoid cancer was diagnosed in 5 patients $(15.6 \%)$, and in 6 patients $(18.8 \%)$ the type of the tumor was unknown. A total of 25 patients (78.1\%) underwent surgical resection of RCC; 16 patients $(50 \%)$ received prior cytokine therapies, including IFN- $\alpha$ and IL-2; the remaining 16 patients had received no prior treatment. An ECOG PS of 0, 1, 2, 3 and 4 was observed in $15,13,2,1$ and 1 patients $(46.9,40.6,6.3,3.1$ and $3.1 \%)$, respectively. Patients with a PS of 3 or 4 were considered suitable to receive chemotherapy, as their poor PS was attributed to pain from bone metastasis or bone fracture. When the MSKCC risk index was calculated, 1 (3.1\%) of the patients was classed as favorable-risk, $23(71.9 \%)$ were intermediate-risk and $8(25.0 \%)$ were poor-risk. When risk was calculated according to the Heng risk group, $1(3.1 \%)$ of the patients were favorable-risk, $20(62.5 \%)$ were intermediate-risk and $11(34.4 \%)$ were poor-risk.

The mean value of the serum CRP level was $3.26 \mathrm{mg} / \mathrm{dl}$ and its range was $0.03-21.15 \mathrm{mg} / \mathrm{dl}$, indicating wide variation among patients. A total of 21 patients $(65.5 \%)$ had an mGPS of $0,3(9.4 \%)$ had an mGPS of 1 , and $8(25.0 \%)$ had an mGPS of 2. A total of 5 patients $(15.6 \%)$ did not receive subsequent therapy, 19 (59.4\%) received up to second-line chemotherapy, and $9(28.1 \%)$ received third- or further-line chemotherapy.

Efficacy. The median PFS of all patients was 212.0 days [95\% confidence interval (CI): 13.0-960] and the median OS was 487.5 days (95\% CI: 30.0-2,137.0).

Correlation analysis of survival and clinical factors. The correlation between survival and clinical parameters was then examined (Table II). A longer PFS and OS were significantly correlated with the MSKCC risk group, the Heng risk group and PS. Clinical parameters corresponding to inflammatory status, including neutrophil/lymphocyte ratio (NLR), serum CRP level, serum albumin level and mGPS, were also significantly associated with PFS and OS. The correlation index of the mGPS was the highest $(\mathrm{R}=0.575, \mathrm{P}=0.001)$ among all of the parameters examined. Receiving subsequent therapies was 
Table I. Patient characteristics $(n=32)$.

\begin{tabular}{ll}
\hline Characteristics & N (\%) \\
\hline
\end{tabular}

Age, years

Mean

65.4

Median (range)

Gender

Male

Female

Histological type

Clear cell carcinoma

$21(65.6)$

Others

$5(15.6)$

Unknown

$6(18.8)$

Resection of the primary lesion

Present

$25(78.1)$

Absent

7 (21.9)

Previous treatment (e.g., cytokines)

Present

$16(50.0)$

Absent

$16(50.0)$

ECOG performance status

0

1

2

3

4

MSKCC risk group

Favorable

Intermediate

Poor

$15(46.9)$

$13(40.6)$

2 (6.3)

$1(3.1)$

$1(3.1)$

$1(3.1)$

$23(71.9)$

$8(25.0)$

Heng risk group

Favorable

$1(3.1)$

Intermediate

$20(62.5)$

$11(34.4)$

WBC $(/ \mu 1)$

Mean

$6,403.1$

Median (range)

C-reactive protein $(\mathrm{mg} / \mathrm{dl})$

Mean

3.26

Median (range)

Albumin (g/dl)

Mean

Median (range)

$\mathrm{LDH}(\mathrm{mg} / \mathrm{dl})$

Mean

Median (range)

Corrected calcium $(\mathrm{mg} / \mathrm{dl})$

Mean

Median (range)

Creatinine $(\mathrm{mg} / \mathrm{dl})$

Mean

Median (range)

Neutrophil/lymphocyte ratio

Mean

5.02

Median (range)
Table I. Continued.

\begin{tabular}{lc}
\hline Characteristics & $\mathrm{N}(\%)$ \\
\hline Modified GPS & $21(65.6)$ \\
0 & $3(9.4)$ \\
1 & $8(25.0)$ \\
2 & \\
Sequential therapy & $5(15.6)$ \\
None & $19(59.4)$ \\
Second-line & $9(28.1)$ \\
Third- or further-line & \\
Progression-free survival (days) & 258 \\
Mean & $212(13.0-960.0)$ \\
Median (range) & 738 \\
Overall survival (days) & \\
Mean & $487.5(30.0-2,137.0)$ \\
Median (range)
\end{tabular}

ECOG, Eastern Cooperative Oncology Group; MSKCC, Memorial Sloan Kettering Cancer Center; WBC, white blood cell; LDH, lactate dehydrogenase; GPS, Glasgow Prognostic Score.

significantly correlated with PFS $(\mathrm{R}=0.437, \mathrm{P}=0.012)$, but not with $\mathrm{OS}(\mathrm{R}=0.273, \mathrm{P}=0.131)$.

Inflammatory conditions and therapeutic effects. Since a correlation of mGPS with survival was demonstrated, PFS and OS were further examined with regard to the mGPS (Fig. 1). The total patients were categorized into one of two groups: Patients with an mGPS of 0 were assigned to the mGPS low group, whereas those with an mGPS of 1 or 2 were assigned to the mGPS high group. The median PFS of the mGPS low group was significantly longer compared with that of the mGPS high group (307.0 vs. 70.0 days, respectively; $\mathrm{P}=0.001)$ based on the Kaplan-Meyer analysis. The median OS of the mGPS low group was also significantly longer compared with that of the mGPS high group (1,081.0 vs. 140.0 days, respectively; $\mathrm{P}=0.001)$. These data suggested that molecular-targeted drugs achieved a more favorable survival in patients of the mGPS low group, who are considered to be of low inflammatory status.

Therapy and inflammatory conditions. In order to assess the reasons for the favorable survival of the mGPS low group, each clinical factor was compared between the two mGPS patient groups (Table III). While all the mGPS low patients had an ECOG PS of 0 or $1(100 \%)$, only $63 \%$ of the mGPS high patients had a PS of 0 or $1(\mathrm{P}<0.038)$. An MSKCC poor risk was observed in $10 \%$ of the mGPS low patients, but in $55 \%$ of mGPS high patients $(\mathrm{P}<0.018)$. In terms of histological diagnosis, based on its molecular mechanisms, clear cell carcinoma is considered to be more sensitive to molecular-targeted drugs compared with tumors of other histological types. Clear cell carcinoma was observed in $86 \%$ of the mGPS low and in $45 \%$ of the mGPS high patients $(\mathrm{P}<0.05)$. These results suggested that mGPS high patients included a population with a poor general condition and drug resistance. Regarding initial 
Table II. Correlation analysis of survival and clinical factors.

\begin{tabular}{|c|c|c|}
\hline Factors & $\begin{array}{l}\text { Progression-free } \\
\text { survival }\end{array}$ & Overall survival \\
\hline Age & $0.247(0.173)$ & $0.218(0.232)$ \\
\hline Gender & $0.059(0.747)$ & $0.063(0.732)$ \\
\hline $\begin{array}{l}\text { Resection of the } \\
\text { primary lesion }\end{array}$ & $0.250(0.168)$ & $0.323(0.071)$ \\
\hline MSKCC risk group & $0.451(0.010)$ & $0.499(0.004)$ \\
\hline Heng risk group & $0.427(0.015)$ & $0.556(0.001)$ \\
\hline Previous treatment & $0.152(0.405)$ & $0.135(0.460)$ \\
\hline $\begin{array}{l}\text { ECOG performance } \\
\text { status }\end{array}$ & $0.523(0.002)$ & $0.554(0.001)$ \\
\hline WBC & $0.264(0.145)$ & $0.261(0.149)$ \\
\hline NLR & $0.531(0.002)$ & $0.533(0.002)$ \\
\hline C-reactive Protein & $0.390(0.027)$ & $0.485(0.005)$ \\
\hline Albumin & $0.427(0.015)$ & $0.613(<0.0001)$ \\
\hline mGPS & $0.575(0.001)$ & $0.591(<0.0001)$ \\
\hline LDH & $0.263(0.146)$ & $0.183(0.315)$ \\
\hline Corrected calcium & $0.135(0.461)$ & $0.065(0.722)$ \\
\hline Creatinine & $0.098(0.592)$ & $0.049(0.788)$ \\
\hline PFS & - & $0.743(<0.0001)$ \\
\hline OS & $0.743(<0.0001)$ & - \\
\hline Sequential therapy & $0.437(0.012)$ & $0.273(0.131)$ \\
\hline
\end{tabular}

Data are presented as $\mathrm{R}$ (P-value). Bold print indicates statistical significance. MSKCC, Memorial Sloan Kettering Cancer Center; ECOG, Eastern Cooperative Oncology Group; WBC, white blood cell; NLR, neutrophil/lymphocyte ratio; mGPS, modified Glasgow Prognostic Score; LDH, lactate dehydrogenase; PFS, progression-free survival; OS, overall survival.

therapy, sunitinib was administered in $57 \%$ of the mGPS low patients and in 54\% of the mGPS high patients $(\mathrm{P}=0.065)$, suggesting no significant differences between the two groups. Although $63.6 \%$ of the mGPS high patients received initial therapy alone, only $5 \%$ of the mGPS low patients received initial therapy alone. The percentage of patients who received second-line therapy in the mGPS low and mGPS high groups was 72 and $36 \%$, respectively, and the percentage of patients who received third- or further-line therapy was 33 and $18 \%$, respectively $(\mathrm{P}=0.003)$.

\section{Discussion}

The pathogenesis of RCC has been considered to be closely associated with inflammation. A variety of indices related to inflammation have been reported to exhibit a predictive value for RCC, including serum CRP level, platelet count, NLR $(31,32)$, platelet to lymphocyte ratio $(31,33)$, lymphocyte-to-monocyte ratio $(31,34)$, the prognostic nutritional index $(31,35)$, GPS (29) and mGPS (27). Cytokines and their receptors, such as IL-6 and granulocyte-stimulating factor receptor 1 have also been reported as indices of inflammatory status. Serum CRP and albumin levels were found to be particularly good indicators of inflammatory status. A retrospective evaluation of 406 RCC
Table III. Therapy and inflammatory status of the mGPS low and high patient groups.

\begin{tabular}{|c|c|c|c|c|c|}
\hline \multirow[b]{2}{*}{ Variables } & \multicolumn{2}{|c|}{ mGPS low } & \multicolumn{2}{|c|}{ mGPS high } & \multirow[b]{2}{*}{ P-value } \\
\hline & $\mathrm{n}=21$ & $(\%)$ & $\mathrm{n}=11$ & $(\%)$ & \\
\hline ECOG PS & & & & & $0.038^{\mathrm{a}}$ \\
\hline 0 & 12 & 57 & 3 & 27 & \\
\hline 1 & 9 & 43 & 4 & 36 & \\
\hline 2 & 0 & 0 & 2 & 18 & \\
\hline 3 & 0 & 0 & 1 & 9 & \\
\hline 4 & 0 & 0 & 1 & 9 & \\
\hline MSKCC & & & & & $0.018^{\mathrm{b}}$ \\
\hline Favorable & 1 & 4 & 0 & 0 & \\
\hline Intermediate & 18 & 86 & 5 & 45 & \\
\hline Poor & 2 & 10 & 6 & 55 & \\
\hline Histology & & & & & $0.018^{\mathrm{b}}$ \\
\hline $\mathrm{CC}$ & 18 & 86 & 5 & 45 & \\
\hline $\mathrm{pRCC}$ & 0 & 0 & 3 & 27 & \\
\hline Others & 3 & 14 & 3 & 27 & \\
\hline Therapeutic drugs & & & & & $0.0647^{\mathrm{b}}$ \\
\hline Sunitinib & 12 & 57 & 6 & 54 & \\
\hline Sorefenib & 3 & 14 & 1 & 5 & \\
\hline Pazopanib & 5 & 24 & 2 & 10 & \\
\hline Temsirolimus & 1 & 5 & 2 & 10 & \\
\hline Subsequent therapy ${ }^{c}$ & & & & & $0.003^{b}$ \\
\hline None & 1 & 5 & 7 & 64 & \\
\hline Second-line & 15 & 72 & 4 & 36 & \\
\hline Third or further-line & 7 & 33 & 2 & 18 & \\
\hline
\end{tabular}

${ }^{a}$ Wilcoxon rank sum test, ${ }^{b}$ Pearson's Chi-square test. 'None, number of patients, who completed first-line chemotherapy alone, or who are currently receiving first-line therapy; second-line, number of patients who received second-line chemotherapy, or who are currently treated with second-line chemotherapy; third- or further-line, number of patients, who received third- or further-line chemotherapy, or who are currently treated with third- or further-line chemotherapy. Based on the definition of each item, 2 patients are repeatedly counted in both the mGPS low and the mGPS high groups. mGPS, modified Glasgow Prognostic Score; ECOG PS, Eastern Cooperative Oncology Group performance status; MSKCC, Memorial Sloan Kettering Cancer Center; CC, clear cell; pRCC, papillary renal cell carcinoma.

patients indicated that the $\mathrm{CRP} /$ albumin ratio was valuable for the prediction of OS (31). The CRP/albumin ratio was also found to be an independent prognostic factor in hepatocellular (36) and esophageal carcinoma (37).

The present study investigated the mGPS of 32 RCC patients prior to the initial chemotherapy, and the mGPS low patients (score 0 ) exhibited a favorable prognosis. mGPS is also calculated using serum CRP and albumin levels. Lamb et al reported the predictive value of mGPS based on analyses of 169 RCC patients who received curative resection (27). Previous studies that assessed the predictive value of mGPS were mostly targeted towards the curability of RCC patients who had undergone curative resection. Therefore, there is 

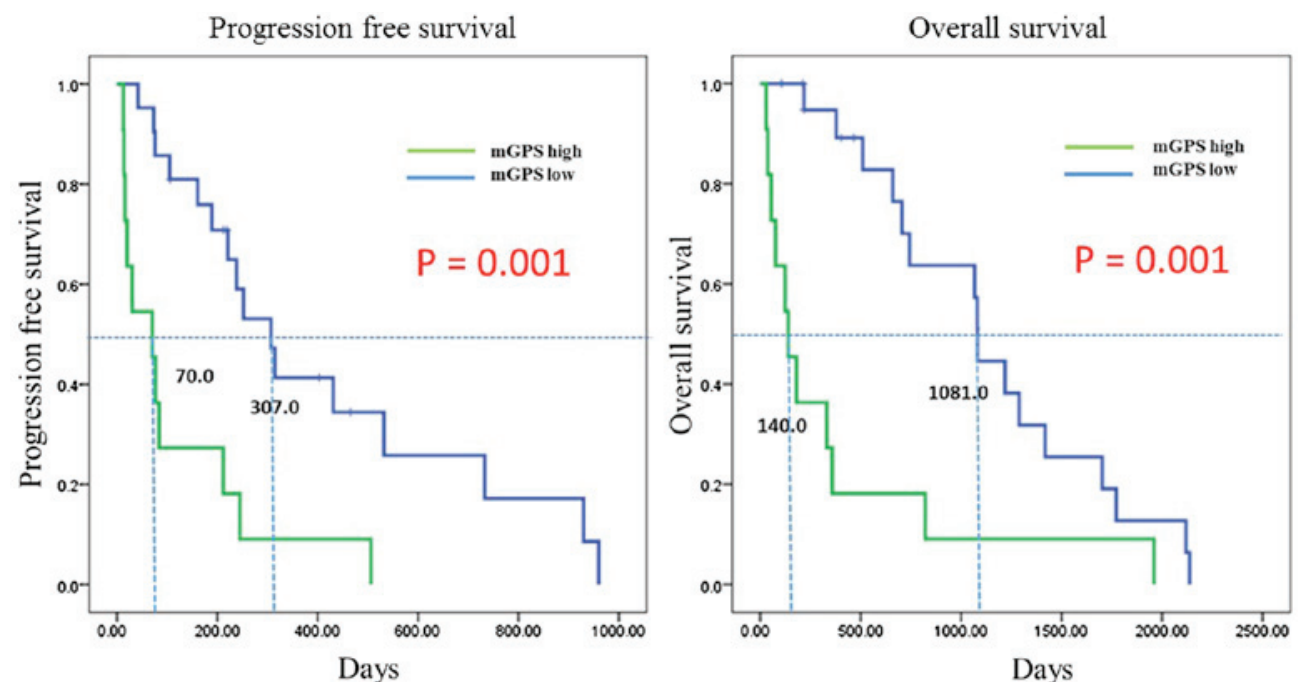

Figure 1. Kaplan-Meier curves of progression-free survival (PFS) and overall survival (OS) of mGPS low and mGPS high patients. PFS and OS were estimated using log-rank analysis. mGPS, modified Glasgow Prognostic Score. The vertical bars show censored cases. The numbers 70, 307, 140 and 1081 indicate the median number of days for the indicated groups.

little information available regarding the predictive value of the mGPS for metastatic or recurrent RCC patients who were treated with molecular-targeted agents. The present findings, indicating a positive correlation between PFS and OS of these RCC patients and their mGPS, strongly suggest a predictive value of mGPS for patients with metastatic or recurrent RCC.

The mechanisms underlying the correlation of inflammatory status with prognosis of metastatic or recurrent RCC have been considered to be as follows: First, since inflammation-associated cytokines may directly induce tumor cell growth, there may be a higher induction of RCC cell proliferation in mGPS high patients compared with that in mGPS low patients. Second, inflammation is often associated with cachexia and deterioration of the patients' general condition, resulting in insufficient administration of chemotherapy. In this study, favorable PFS with first-line chemotherapy in mGPS low patients suggested that the mGPS may be correlated with efficacy of the initial molecular-targeted therapy. One possible reason for this correlation is that the mGPS low patients may have harbored cancer cells with relatively non-aggressive characteristics (low proliferation rate, as mentioned above) that were sensitive to tyrosine kinase inhibitors (TKIs). The cell proliferation of clear cell carcinoma has been reported to depend on vascular endothelial growth factor receptor (VEGFR) signaling and is sensitive to TKIs, which inhibits the effect of VEGFR signaling (7-10). However, the serum CRP level in patients with clear cell carcinoma was found to be higher compared with that in patients with papillary cell carcinoma (38). Although the mGPS low patient group included a higher percentage of clear cell carcinoma patients than the mGPS high patient group in this study, it is not conclusive that the favorable PFS of the mGPS low patients is attributed to the high percentage of clear cell carcinoma patients. The present data also demonstrated that the mGPS low patients tended to exhibit favorable PS and MSKCC. Parameters such as PS and MSKCC are considered to reflect the patients' general status; thus, the longer PFS of the mGPS low patients may also be associated with their better general condition due to a low inflammatory status.

Additionally, the mGPS low patients exhibited longer OS compared with the mGPS high patients. This difference may be associated with the high percentage of patients who were able to receive subsequent chemotherapies. Overall survival following termination of the initial chemotherapy in the mGPS low patients was found to be significantly longer compared with that of the mGPS high patients (median OS, 1,111 days vs. 180 days, respectively; $\mathrm{P}=0.006$ ). Although no examination of the mGPS of each patient group after termination of the initial chemotherapy was performed, this finding suggested that subsequent chemotherapy may also be effective for mGPS low patients with metastatic and recurrent RCC.

The molecular-targeted drugs that may be suitable for mGPS high patients with metastatic or recurrent RCC must be determined. For this purpose, an understanding of the regulatory mechanisms of inflammation in RCC patients is required. Mammalian target of rapamycin (mTOR) inhibitor suppresses cellular growth of RCC by blocking mTOR-mediated signaling. Cytokine signaling pathways including IL-6, tumor growth factor- $\beta$ and insulin-like growth factor share the common property of mTOR activation $(39,40)$. Since inhibition of mTOR has been demonstrated to induce muscle protein wasting in cancer cachexia $(41,42)$, it may be suggested that an mTOR inhibitor may be suitable only for mGPS low patients. However, inflammatory cytokines have been reported to suppress the production of VEGF from tumor cells and to regulate the tumor microenvironment (43). Although a direct correlation between inflammatory status and the efficacy of anti-angiogenic therapy has not been reported, molecular-targeted drugs and inflammatory cytokines may exert synergistic effects, and further investigation is required to determine the appropriate therapeutic strategy for RCC patients with a high inflammatory status.

Although the present study was performed in order to retrospectively investigate a small number of patients, superiority of PFS and OS of the mGPS low over the mGPS high 
patient group was observed. These results are consistent with results from curatively resected RCC patients, and the potential prognostic value of mGPS for metastatic or recurrent RCC patients treated with molecular-targeted agents may be a novel finding.

The present study strongly suggested that inflammatory status prior to the initial chemotherapy is correlated with PFS and OS. Since the possible reasons for the favorable survival of patients with low inflammatory status may include acceleration of tumor growth by inflammation and insufficient administration of chemotherapies due to inflammation, the selection of the appropriate molecular-targeted drugs should be further investigated. Inflammation-related indices, such as the mGPS, reflect the interaction between the host immune system and the tumor. The mGPS may be used not only as a predictive parameter of RCC, but also as a biomarker to monitor the actual inflammatory status in cases of future treatments by immune checkpoint inhibitors.

\section{Acknowledgements}

The authors would like to thank the medical staff of each institution who contributed to the treatment of the patients.

\section{References}

1. Jemal A, Bray F, Center MM, Ferlay J, Ward E and Forman D: Global cancer statistics. CA Cancer J Clin 61: 69-90, 2011.

2. Naito S, Tomita Y, Rha SY, Uemura H, Oya M, Song HZ, Zhong LH and Wahid MI: Kidney Cancer Working Group report. Jpn J Clin Oncol 40 (Suppl 1): i51-i56, 2010.

3. Siegel R, Naishadham D and Jemal A: Cancer statistics, 2013. CA Cancer J Clin 63: 11-30, 2013.

4. MacLennan S, Imamura M, Lapitan MC, Omar MI, Lam TB, Hilvano-Cabungcal AM, Royle P, Stewart F, MacLennan G, MacLennan SJ, et al: Systematic review of oncological outcomes following surgical management of localised renal cancer. Eur Urol 61: 972-993, 2012.

5. Dutcher JP, Fisher RI, Weiss G, Aronson F, Margolin K, Louie A, Mier J, Caliendo G, Sosman JA, Eckardt JR, et al: Outpatient subcutaneous interleukin-2 and interferon-alpha for metastatic renal cell cancer: Five-year follow-up of the cytokine working group study. Cancer J Sci Am 3: 157-162, 1997.

6. Negrier S, Escudier B, Lasset C, Douillard JY, Savary J, Chevreau C, Ravaud A, Mercatello A, Peny J, Mousseau M, et al: Recombinant human interleukin-2, recombinant human interferon alfa-2a, or both in metastatic renal-cell carcinoma. Groupe Francais d'Immunotherapie. N Engl J Med 338: 1272-1278, 1998.

7. Motzer RJ, Hutson TE, Cella D, Reeves J, Hawkins R, Guo J, Nathan P, Staehler M, de Souza P, Merchan JR, et al: Pazopanib versus sunitinib in metastatic renal-cell carcinoma. N Engl J Med 369: 722-731, 2013.

8. Motzer RJ, Hutson TE, Tomczak P, Michaelson MD, Bukowski RM, Rixe O, Oudard S, Negrier S, Szczylik C, Kim ST, et al: Sunitinib versus interferon alfa in metastatic renal-cell carcinoma. N Engl J Med 356: 115-124, 2007.

9. Sternberg CN, Davis ID, Mardiak J, Szczylik C, Lee E, Wagstaff J, Barrios CH, Salman P, Gladkov OA, Kavina A, et al: Pazopanib in locally advanced or metastatic renal cell carcinoma: Results of a randomized phase III trial. J Clin Oncol 28: 1061-1068, 2010.

10. Motzer RJ, Barrios CH, Kim TM, Falcon S, Cosgriff T, Harker WG, Srimuninnimit V, Pittman K, Sabbatini R, Rha SY, et al: Phase II randomized trial comparing sequential first-line everolimus and second-line sunitinib versus first-line sunitinib and second-line everolimus in patients with metastatic renal cell carcinoma. J Clin Oncol 32: 2765-2772, 2014.

11. Motzer RJ, Bacik J, Murphy BA, Russo P and Mazumdar M: Interferon-alfa as a comparative treatment for clinical trials of new therapies against advanced renal cell carcinoma. J Clin Oncol 20: 289-296, 2002.
12. Heng DY, Xie W, Regan MM, Harshman LC, Bjarnason GA Vaishampayan UN, Mackenzie M, Wood L, Donskov F, Tan MH, et al: External validation and comparison with other models of the international metastatic renal-cell carcinoma database consortium prognostic model: A population-based study. Lancet Oncol 14: 141-148, 2013.

13. Heng DY, Xie W, Regan MM, Warren MA, Golshayan AR, Sahi C, Eigl BJ, Ruether JD, Cheng T, North S, et al: Prognostic factors for overall survival in patients with metastatic renal cell carcinoma treated with vascular endothelial growth factor-targeted agents: Results from a large, multicenter study. J Clin Oncol 27: 5794-5799, 2009.

14. Bromwich EJ, McArdle PA, Canna K, McMillan DC, McNicol AM, Brown M and Aitchison M: The relationship between T-lymphocyte infiltration, stage, tumour grade and survival in patients undergoing curative surgery for renal cell cancer. Br J Cancer 89: 1906-1908, 2003.

15. Crumley AB, McMillan DC, McKernan M, McDonald AC and Stuart RC: Evaluation of an inflammation-based prognostic score in patients with inoperable gastro-oesophageal cancer. Br J Cancer 94: 637-641, 2006.

16. Forrest LM, McMillan DC, McArdle CS, Angerson WJ and Dunlop DJ: Evaluation of cumulative prognostic scores based on the systemic inflammatory response in patients with inoperable non-small-cell lung cancer. Br J Cancer 89: 1028-1030, 2003.

17. Fujikawa K, Matsui Y, Oka H, Fukuzawa S and Takeuchi H: Serum C-reactive protein level and the impact of cytoreductive surgery in patients with metastatic renal cell carcinoma. J Urol 162: 1934-1937, 1999.

18. Bazzi WM, Tin AL, Sjoberg DD, Bernstein M and Russo P: The prognostic utility of preoperative neutrophil-to-lymphocyte ratio in localized clear cell renal cell carcinoma. Can J Urol 23: 8151-8154, 2016.

19. Hu K, Lou L, Ye J and Zhang S: Prognostic role of the neutrophil-lymphocyte ratio in renal cell carcinoma: A meta-analysis. BMJ Open 5: e006404, 2015

20. Templeton AJ, Knox JJ, Lin X, Simantov R, Xie W, Lawrence N, Broom R, Fay AP, Rini B, Donskov F, et al: Change in neutrophil-to-lymphocyte ratio in response to targeted therapy for metastatic renal cell carcinoma as a prognosticator and biomarker of efficacy. Eur Urol 70: 358-364, 2016.

21. Zhang GM, Zhu Y, Gu WJ, Zhang HL, Shi GH and Ye DW: Pretreatment neutrophil-to-lymphocyte ratio predicts prognosis in patients with metastatic renal cell carcinoma receiving targeted therapy. Int J Clin Oncol 21: 373-378, 2016.

22. Argiles JM, Busquets S, Stemmler B and López-Soriano FJ: Cancer cachexia: Understanding the molecular basis. Nat Rev Cancer 14: 754-762, 2014.

23. McMillan DC, Scott HR, Watson WS, Preston T, Milroy R and McArdle CS: Longitudinal study of body cell mass depletion and the inflammatory response in cancer patients. Nutr Cancer 31: 101-105, 1998.

24. O'Gorman P, McMillan DC and McArdle CS: Prognostic factors in advanced gastrointestinal cancer patients with weight loss. Nutr Cancer 37: 36-40, 2000.

25. Scott HR, McMillan DC, Crilly A, McArdle CS and Milroy R: The relationship between weight loss and interleukin 6 in non-small-cell lung cancer. Br J Cancer 73: 1560-1562, 1996.

26. Scott HR, McMillan DC, Forrest LM, Brown DJ, McArdle CS and Milroy R: The systemic inflammatory response, weight loss, performance status and survival in patients with inoperable non-small cell lung cancer. Br J Cancer 87: 264-267, 2002.

27. Lamb GW, Aitchison M, Ramsey S, Housley SL and McMillan DC: Clinical utility of the Glasgow Prognostic Score in patients undergoing curative nephrectomy for renal clear cell cancer: Basis of new prognostic scoring systems. Br J Cancer 106: 279-283, 2012.

28. Proctor MJ, Morrison DS, Talwar D, Balmer SM, O'Reilly DS, Foulis AK, Horgan PG and McMillan DC: An inflammation-based prognostic score (mGPS) predicts cancer survival independent of tumour site: A Glasgow inflammation outcome study. Br J Cancer 104: 726-734, 2011.

29. Ramsey S, Lamb GW, Aitchison M, Graham J and McMillan DC: Evaluation of an inflammation-based prognostic score in patients with metastatic renal cancer. Cancer 109: 205-212, 2007.

30. McMillan DC, Crozier JE, Canna K, Angerson WJ and McArdle CS: Evaluation of an inflammation-based prognostic score (GPS) in patients undergoing resection for colon and rectal cancer. Int J Colorectal Dis 22: 881-886, 2007. 
31. Chen Z, Shao Y, Fan M, Zhuang Q, Wang K, Cao W, Xu X and $\mathrm{He} X$ : Prognostic significance of preoperative C-reactive protein: Albumin ratio in patients with clear cell renal cell carcinoma. Int J Clin Exp Pathol 8: 14893-14900, 2015.

32. Pichler M, Hutterer GC, Stoeckigt C, Chromecki TF, Stojakovic T, Golbeck S, Eberhard K, Gerger A, Mannweiler S, Pummer K and Zigeuner R: Validation of the pre-treatment neutrophil-lymphocyte ratio as a prognostic factor in a large European cohort of renal cell carcinoma patients. Br J Cancer 108: 901-907, 2013.

33. Gunduz S, Mutlu H, Tural D, Yildiz Ö, Uysal M, Coskun HS and Bozcuk $\mathrm{H}$ : Platelet to lymphocyte ratio as a new prognostic for patients with metastatic renal cell cancer. Asia Pac J Clin Oncol 11: 288-292, 2015

34. Chang Y, An H, Xu L, Zhu Y, Yang Y, Lin Z and Xu J: Systemic inflammation score predicts postoperative prognosis of patients with clear-cell renal cell carcinoma. Br J Cancer 113: 626-633, 2015.

35. Jeon HG, Choi DK, Sung HH, Jeong BC, Seo SI, Jeon SS, Choi HY and Lee HM: Preoperative prognostic nutritional index is a significant predictor of survival in renal cell carcinoma patients undergoing nephrectomy. Ann Surg Oncol 23: 321-327, 2016.

36. Kinoshita A, Onoda H, Imai N, Iwaku A, Oishi M, Tanaka K, Fushiya N, Koike K, Nishino $\mathrm{H}$ and Matsushima M: The C-reactive protein/albumin ratio, a novel inflammation-based prognostic score, predicts outcomes in patients with hepatocellular carcinoma. Ann Surg Oncol 22: 803-810, 2015.

37. Wei XL, Wang FH, Zhang DS, Qiu MZ, Ren C, Jin Y, Zhou YX, Wang DS, He MM, Bai L, et al: A novel inflammation-based prognostic score in esophageal squamous cell carcinoma: The C-reactive protein/albumin ratio. BMC Cancer 15: 350, 2015.
38. Jabs WJ, Busse M, Krüger S, Jocham D, Steinhoff J and Doehn C: Expression of C-reactive protein by renal cell carcinomas and unaffected surrounding renal tissue. Kidney Int 68: 2103-2110, 2005.

39. Robert F, Mills JR, Agenor A, Wang D, DiMarco S, Cencic R, Tremblay ML, Gallouzi IE, Hekimi S, Wing SS and Pelletier J: Targeting protein synthesis in a Myc/mTOR-driven model of anorexia-cachexia syndrome delays its onset and prolongs survival. Cancer Res 72: 747-756, 2012.

40. Yoshida T, Tabony AM, Galvez S, Mitch WE, Higashi Y, Sukhanov S and Delafontaine P: Molecular mechanisms and signaling pathways of angiotensin II-induced muscle wasting: Potential therapeutic targets for cardiac cachexia. Int J Biochem Cell Biol 45: 2322-2332, 2013.

41. Gordon BS, Kelleher AR and Kimball SR: Regulation of muscle protein synthesis and the effects of catabolic states. Int J Biochem Cell Biol 45: 2147-2157, 2013.

42. Manne ND, Lima M, Enos RT, Wehner P, Carson JA and Blough E: Altered cardiac muscle mTOR regulation during the progression of cancer cachexia in the ApcMin/+ mouse. Int J Oncol 42: 2134-2140, 2013.

43. Nagineni CN, William A, Cherukuri A, Samuel W, Hooks JJ and Detrick B: Inflammatory cytokines regulate secretion of VEGF and chemokines by human conjunctival fibroblasts: Role in dysfunctional tear syndrome. Cytokine 78: 16-19, 2016. 